Pathologe 2010 · 31:331-332

DOI 10.1007/s00292-010-1349-3

Online publiziert: 13. August 2010

(c) Springer-Verlag 2010

\author{
C. Wittekind \\ Institut für Pathologie, Universitätsklinikum Leipzig
}

\title{
TNM-System 2010
}

\section{Zur 7. Auflage der TNM-Klassifikation maligner Tumoren}

Der Prozess, der im klinisch umgangssprachlichen Gebrauch als „Staging“ bezeichnet wird, beinhaltet die Feststellung und Beschreibung der anatomischen Ausbreitung eines malignen Tumors. Diese Beschreibung schließt die Ausdehnung des Primärtumors (T), die Ausbreitung eines Tumors in den regionären Lymphknotenmetastasen $(\mathrm{N})$ und das eventuelle Vorhandensein von Fernmetastasen ( $\mathrm{M}$ ) ein. $\mathrm{T}, \mathrm{N}$ und $\mathrm{M}$ können sowohl klinisch festgestellt werden (cTNM) als auch durch die pathologisch-anatomische Untersuchung eines Tumorresektates (pTNM). Die klinische TNM-Klassifikation ist wichtig für die Auswahl der Therapie. Die postoperative histologische (pathologische) TNM-Klassifikation wird für die Indikation zur adjuvanten Therapie gebraucht und liefert wichtige Daten, um die Prognose abzuschätzen. Die exakte Feststellung der pTNM-Klassifikation bedeutet für den verantwortlichen $\mathrm{Pa}$ thologen eine besondere Verantwortung, nicht nur durch eine genaue makroskopische und mikroskopische Untersuchung die Parameter für eine Klassifikation festzulegen, sondern auch, diese Parameter in ein korrektes TNM-Klassifikationsschema einzubringen. Diese verantwortungsvolle Tätigkeit benötigt eine stetige Befassung mit der TNM-Klassifikation und ihren Änderungen, die von Zeit zu Zeit vorgenommen werden.

Die 5. Auflage der TNM-Klassifikation maligner Tumoren wurde 1997 publiziert, die 6. Auflage 2002. Dieses Intervall von 5 Jahren wurde von denjenigen, die sich mit der Validierung der bestehenden
Definitionen und ihrer Weiterentwicklung beschäftigen, als zu kurz befunden, um in diesem Zeitraum Daten - insbesondere zum Verlauf von Patienten mit malignen Tumoren - zu sammeln. Deswegen wurde die 7 . Auflage der TNMKlassifikation 7 Jahre nach der 6. Auflage veröffentlicht, um Interessierten Gelegenheit zu geben, Daten zu publizieren, die die Vorschläge der 6. Auflage untermauerten oder widerlegten bzw. Modifikationen vorschlugen.

An den Definitionen der 6. Auflage wurde zum Teil heftige Kritik geübt. Diese stützte sich auf mangelnde Absprachen, zu geringe Berücksichtigung von laufenden Studien und eine nicht angebrachte Betrachtung tumorbiologischer Probleme. Eine aktive Konsensussuche wurde von den „Task Forces“ des „American Joint Committee on Cancer" (AJCC) nicht betrieben. Die Kritiker wurden aufgefordert, die von ihnen angemahnten Schwachpunkte durch eigene Daten zu unterlegen. Dieses ist nur zum Teil erfolgt. Um die Kritik einer mitunter willkürlichen Veränderung von TNM-Definitionen zu widerlegen, wurde nach der Publikation der 6. Auflage von der „Union Internationale Contre le Cancer" (UICC) ein Verfahren institutionalisiert, um Vorschläge zur Verbesserung der TNM-Klassifikation zu evaluieren. Die Vorschläge der Autoren und die Ergebnisse der Literatursuche werden durch ein Expertenpanel der UICC und durch die Mitglieder des „TNM Prognostic Factors Project Committee" ausgewertet. Detaillierte Angaben und eine Checkliste, die die Formulierung von Änderungsvorschlägen erleichtern soll, können über die Webseite der UICC bezogen werden (www.uicc.org).

Mit der Vorstellung der 7. Auflage sollte dieser Prozess noch weiter verfeinert und ausgebaut werden. Beim Vergleich der 7 Auflagen von AJCC und UICC ergaben sich zahlreiche Unterschiede, die auf der Basis einer fehlenden Kommunikation von Seiten des AJCC zustande gekommen sind. Alle am TNM-System Interessierten sind aufgefordert, unmittelbar nach Publikation der 7. Auflage Daten zu sammeln, um alte und neue Definitionen des TNMSystems auf ihre Wertigkeit zu überprüfen. Es ist zu hoffen, dass nach 7 Jahren bis zur voraussichtlichen Publikation der nächsten Auflage genügend Daten vorliegen, um die Definitionen in der 8. Auflage zu modifizieren, zu ergänzen oder zu erneuern. Ein besonderer Schwerpunkt soll dabei auf der Integration neuer molekularer Daten in das TNM-System liegen. Erste Schritte, nicht nur anatomische Parameter mit einzubeziehen, wurden mit der Berücksichtigung von Tumormarkern bei der Stadiengruppierung von Hodentumoren gemacht und in einem neuen Ansatz in der 7. Auflage durch die Trennung von anatomischer Stadiengruppierung und prognostischen Gruppen fortgeführt. In diesen neuen prognostischen Gruppierungen wurden neben anatomischen Parametern Grad und Lokalisation (bei Ösophagustumoren) sowie PSAWert und Gleason-Grad (beim Prostatakarzinom) berücksichtigt. Es wird Aufgabe der weltweiten TNM-Gemeinde sein, für die nächste Auflage weitere Parame- 
ter zu evaluieren und sinnvoll in Vorschläge für eine Weiterentwicklung der TNMKlassifikation einzubringen.

In diesem Heft von Der Pathologe sollen für die Tumoren der einzelnen Organsysteme die Änderungen und Neuerungen vorgestellt, zum Teil begründet und in ihrer Wertigkeit hinsichtlich der Anwendung kommentiert werden. Weiter soll auf Abschnitte aufmerksam gemacht werden, in denen Bedarf nach Verbesserung und Weiterentwicklung besteht. $\mathrm{Zu}$ dem soll auf Lücken hingewiesen werden, die die Notwendigkeit neuer Klassifikationen deutlich machen.

Ich danke allen Autoren, die sich dieser Aufgabe unterzogen und ihre wichtigen Beiträge für Der Pathologe geschrieben haben.

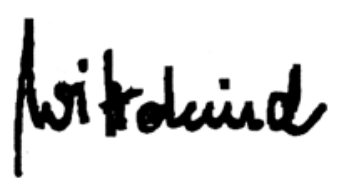

Christian Wittekind

\section{Korrespondenzadresse \\ Prof. Dr. C. Wittekind}

Institut für Pathologie, Universitäts-

klinikum Leipzig

Liebigstr. 26, 04103 Leipzig

wittc@medizin.uni-leipzig.de

\section{Jens Hollmann \\ Führungskompetenz für \\ Leitende Ärzte}

Motivation, Teamführung, Konflikt-

management im Krankenhaus

Heidelberg: Springer 2010, 199 S., 30 Abb., (ISBN 978-3-642-05264-4), 44.95 EUR

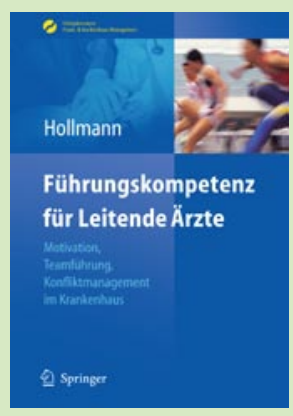

Das Buch vermittelt einen umfassenden und zugleich detailliert-fundierten Ein- und Überblick zu zentralen Themenbereichen ärztlichen Führungshandelns in der Klinik. Der Autor Jens Hollmann hat die Herausforderungen sauber herausgearbeitet. Die Entwicklung und das Führen eines Teams mit Blick auf die sich ständig verändernden Anforderungen an die Zukunftsfähigkeit von Kliniken einerseits sowie die Ausbildung und Motivation junger Kollegen anderseits werden im Klinikalltag oft beiseite gedrängt. Die Bereitschaft, Konflikte als solche zu erkennen und diese aktiv anzugehen, ist eine Kultur, die in Kliniken wenig entwickelt ist. Die ehemals stark autoritär geprägte Führungskultur hat ihre Spuren hinterlassen: Im gegenwärtigen Wettbewerb ist es schwer geworden, engagierte und verantwortliche Mitarbeiter zu finden. Die Professionalisierung in der Mitarbeiterführung wird somit zu einer entscheidenden Variablen im Überleben einer Abteilung oder einer Klinik. Das Buch richtet sich bewusst an alle leitenden Ärzte, die ihre Führungskompetenz erarbeiten müssen. Sie fällt nicht mehr wie früher als Heiligenschein auf den Kopf des Chefs. Beeindruckt hat mich die Lebendigkeit der Beispiele aus dem Klinikalltag, die das Buch nicht nur kurzweilig gestalten, sondern auch den unmittelbaren Zugang zu den dahinterstehenden arbeitspsychologischen Thesen eröffnen. Mir persönlich haben sich durch die Analyse der beispielhaften Situationen, die jede für sich treffsicher die Klinikrealität widerspiegeln, vollkommen neue Perspektiven auf meinen Arbeitsalltag eröffnet. Die Checklisten und Tests, die die theoretischen Ausführungen sehr sinnvoll ergänzen, sind als Denkanstöße und als Grundlage eines bewussteren Führens gut geeignet. Jens Hollmann ist es gelungen, den klinischen Alltag und die arbeitspsychologische Perspektive zu praktisch verwertbaren Wissens-Clustern zu modellieren. Ein tolles Standardwerk hoher didaktischer und sprachlicher Güte mit besonderer Klinikrelevanz. Das Buch trifft wirklich eine Lücke.

M. Schmidt (Bad Bergzabern) 